\title{
Local Blinking HDR LCD Systems for Fast MPRT With High Brightness LCDs
}

\author{
Lin-Yao Liao, Chih-Wei Chen, and Yi-Pai Huang
}

\begin{abstract}
A new impulse-type display named Local Blinking High Dynamic Range (HDR) LCD System was proposed, which utilized local dimming technology to achieve a local blinking effect that not only suppressed motion blur and reduce Moving Picture Response Time (MPRT) to $3.89 \mathrm{~ms}$ but also applied local image compensation to maintain image brightness and details without substituting high power light source. In addition, the intrinsic advantages, such as high dynamic range (high contrast ratio) and low power consumption were also achieved.
\end{abstract}

Index Terms-High-dynamic-range (HDR), local blinking, motion blur, Moving Picture Response Time (MPRT).

\section{INTRODUCTION}

A LIQUID CRYSTAL display (LCD) has been gradually applied to many areas, and LCD performance has to be an important research. However, the quality of moving images has been limited by slow liquid crystal (LC) response and Hold-type driving of LCDs [1], which results in edge-blurring on moving images when human eyes are following an object. This phenomenon has also been named "Motion Blur" [2], which is illustrated in Fig. 1, showing the difference between a static bar and a moving bar with a blurred edge.

To suppress Motion Blur phenomenon, many prior arts have been proposed. One of which is Over-drive [3]-[6], which utilized the driving method to shorten LC response time. The second mainstream approach is high frame rate technology. This technology uses 120/240 Hz motion-estimation/motioncompensation (ME/MC) [7], [8] to drive a higher frame rate rather than conventional $60 \mathrm{~Hz}$ in order to insert a compensated image for smoother moving images. Although these two methods have significantly suppressed Motion Blur phenomenon, slow $\mathrm{LC}$ response time is still an issue as the frame rate is driven higher and higher, a second issue is higher data rate and more complicated IC circuit. An alternative method is Impulse Drive, such as Blinking Backlight and Scanning Backlight [9], [10], which utilized backlight (BL) driving to approach an Impulse-type display, but the main issue of brightness suppression is solved by the substitution of a high power light source to maintain image brightness. For example, Fig. 2 shows a Blinking Backlight system operating full-off and full-on

Manuscript received October 20, 2009; revised December 21, 2009. Current version published March 31, 2010. This work was supported by National Science Council, Taiwan, under Contract NSC 96-2221-E-009-113-MY.

The authors are with the Department of Photonics \& Institute of Electro-Optical Engineering/Display Institute, National Chiao Tung University, Hsinchu 300, Taiwan (e-mail: finalhome.eo95g@nctu.edu.tw).

Color versions of one or more of the figures in this paper are available online at http://ieeexplore.iee.org.

Digital Object Identifier 10.1109/JDT.2009.2039852

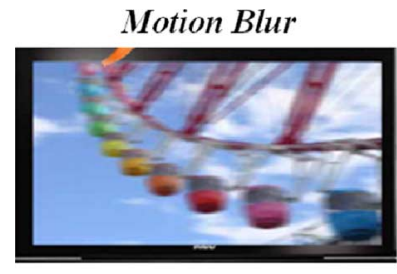

(a)

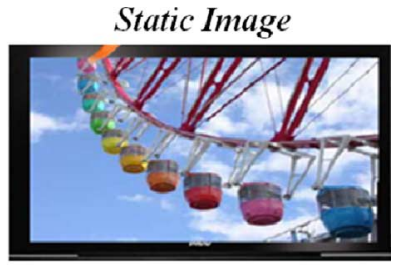

(b)
Fig. 1. (a) Moving image with "Motion Blur" and (b) the static image. [22].

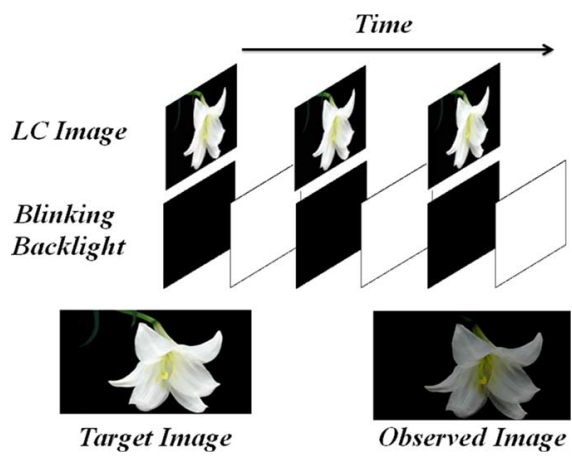

Fig. 2. Blinking Backlight system.

BL frames in one image frame time to achieve Impulse-type display. Although this kind of backlight driving can effectively reduce the observed LC transition time and achieve adequate performance in suppressing Motion Blur, the reducing image brightness was an issue.

To achieve a low motion blur and high brightness performance, a new impulse-type display system named Local Blinking High Dynamic Range (HDR) [11]-[21] LCD System was proposed, which utilized the local dimming technology to achieve a local blinking effect and performed local image compensation to maintain image brightness. In this study, Moving Picture Response Time (MPRT) [22] can be reduced as well as maintaining image brightness without substituting the high power light source.

\section{LOCAL BLINKING HDR LCD SYSTEM}

The main concept of Local Blinking HDR LCD System is to operate a dimming $\mathrm{BL}$ frame and a boosting $\mathrm{BL}$ frame within one image frame time, as shown in Fig. 3(a), the BL frames are determined according to original $\mathrm{BL}$ signals from conventional HDR LCDs, as illustrated in Fig. 3(b). Through BL operation, a local blinking effect was yielded. By combining a LC image compensated according to the integral distribution of these BL frames, an image with the target brightness was displayed. The main benefit of local blinking is that the blinking 


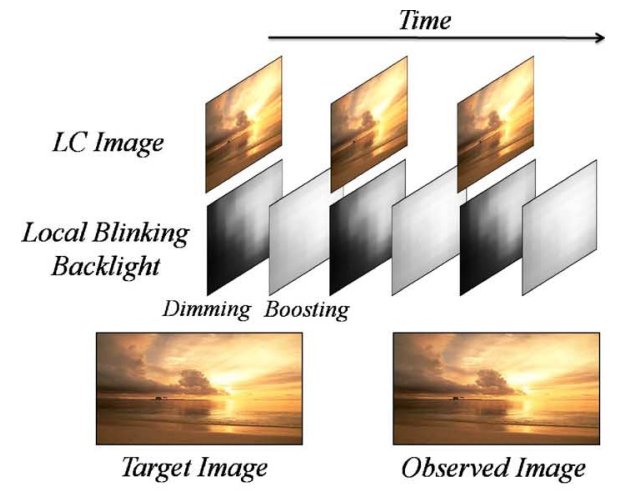

(a)

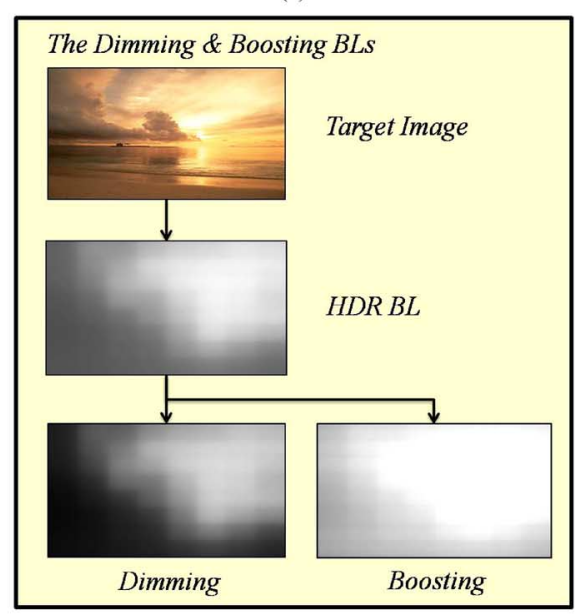

(b)

Fig. 3. (a) The proposed Local Blinking HDR LCD System, and (b) the dimming and boosting BL frames.

operation achieves an Impulse-type display which significantly suppress motion blur, and utilizes the local image compensation to maintain image brightness and details.

The flow chart of the Local Blinking HDR LCD System is shown in Fig. 4(a), and is illustrated as follows.

1) BL signals were calculated according to the target image. Inverse of Mapping Function (IMF) algorithm [23] was used to optimize the BL signals frame by frame and determined the original BL signals.

2) Two BL mapping curves, as shown in Fig. 4(b), are used to determine dimming and boosting BL signals. The dimming and boosting BL mapping curves are derived by the $N$ th power and the $N$ th roots of the normalized BL value, as shown in (1), the larger $N$-value is then applied, and a larger contrast between dimming and boosting BL signals are yielded

$$
\left\{\begin{array}{l}
B L_{\text {Dimming }}=255 \cdot\left(\frac{B L}{255}\right)^{N} \\
B L_{\text {Boosting }}=255 \cdot\left(\frac{B L}{255}\right)^{1 / N} .
\end{array}\right.
$$

3) BL distributions of dimming and boosting BL frames are simulated to represent the human integrated intensity in one image frame.

4) Dividing the target image by the BL distribution and taking the gamma effect $(\gamma)$ of the display device into consider- ation [24], [25], the locally compensated image signals is obtained.

5) Dimming and boosting BL frames with the locally compensated image signals are operated in one image frame, the final image is displayed.

Through the locally compensated image signals with effective local dimming BL distribution of HDR systems, the image brightness and details was compensated locally rather than global image compensation applied in Blinking Backlight system or the substitution of high power light source. Furthermore, this Impulse-type drive also significantly suppressed Motion Blur.

\section{BL MAPPING CURVES AND OPTIMIZATION}

The BL mapping curves, as shown in Fig. 4(b), actually dominates a performance between Motion Blur and image brightness. The larger $N$ is applied, the larger contrast between dimming and boosting curve was yielded, which means the larger $N$-value will generate a more obvious blinking effect to further suppress Motion Blur, but also make the dimming and boosting frames be more similar to conventional Blinking BL operation, which results in reduction of image brightness. In order to obtain a balance performance, the MPRT, which is calculated by $8 \times 8$ normalized blurred edge time (N-BET) [20], and image brightness are investigated by different $N$-values with two driving rate, $60 \mathrm{~Hz} \mathrm{LC}$ with $120 \mathrm{~Hz} \mathrm{BL}$ and $120 \mathrm{~Hz}$ LC with $240 \mathrm{BL}$ frame-rate. The results were also compared with that of different LCD systems, such as conventional LCDs (60 Hz driving), Blinking BL systems $(60 \mathrm{~Hz} \mathrm{LC}$ with $120 \mathrm{~Hz}$

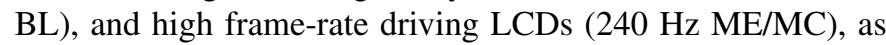
shown in Fig. 5.

Through the results of Local Blinking HDR Systems, the MPRT was further reduced when the $N$-value increased. Although larger $N$-value also leaded to the reduction in image brightness, the $N$-value was chosen as $N=7$ and operated Local Blinking HDR System in $120 \mathrm{~Hz}$ LC with $240 \mathrm{BL}$ frame-rate, MPRT was improved to $3.89 \mathrm{msec}$ and the image brightness was maintained above $90 \%$, which is almost the same as target brightness. For the other impulse drive system, Blinking BL showed a high performance in Motion Blur which the MPRT achieved around $5.5 \mathrm{~ms}$, but the image brightness was half decreased due to the full-blinking driving. In the high frame-rate part, although $240 \mathrm{~Hz} \mathrm{ME/MC}$ technology provided $4.7 \mathrm{~ms}$ MPRT and achieved full image brightness, the issues, such as slow LC response time, high data rate, and complicate IC circuit, still need to be considered, if the frame-rate is driven faster and faster.

\section{Motion Picture And Results}

In order to verify the performance, Fig. 6 shows the simulation results of test image displayed by conventional LCDs (60 $\mathrm{Hz}$ driving), the proposed Local Blinking HDR LCD System $(60 \mathrm{~Hz} \mathrm{LC}+120 \mathrm{~Hz} \mathrm{BL}$ and $120 \mathrm{~Hz} \mathrm{LC}+240 \mathrm{~Hz} \mathrm{BL})$, and Blinking BL systems $(60 \mathrm{~Hz} \mathrm{LC}+120 \mathrm{~Hz} \mathrm{BL}$ and $120 \mathrm{~Hz} \mathrm{LC}+240 \mathrm{~Hz} \mathrm{BL}$ ) to illustrate the performance in Motion Blur and image brightness which the magnified part showed the obvious difference. In the conventional LCD, a serious blurred edge on the petals was observed due to the in- 


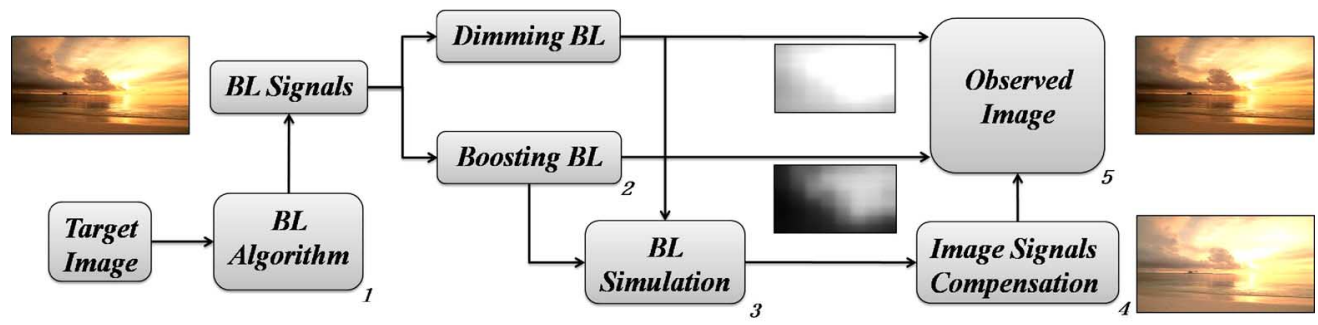

(a)

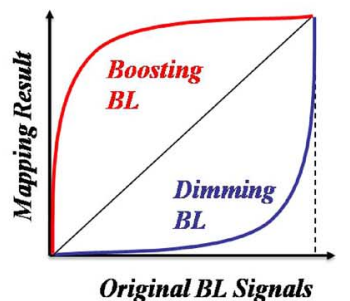

$\left\{\begin{array}{l}B L_{\text {Dimming }}=255 \cdot\left(\frac{B L}{255}\right)^{N} \\ B L_{\text {Boosting }}=255 \cdot\left(\frac{B L}{255}\right)^{1 / N}\end{array}\right.$

(b)

Fig. 4. (a)Flow chart of Local Blinking HDR LCD System, and (b) the BL mapping curves.

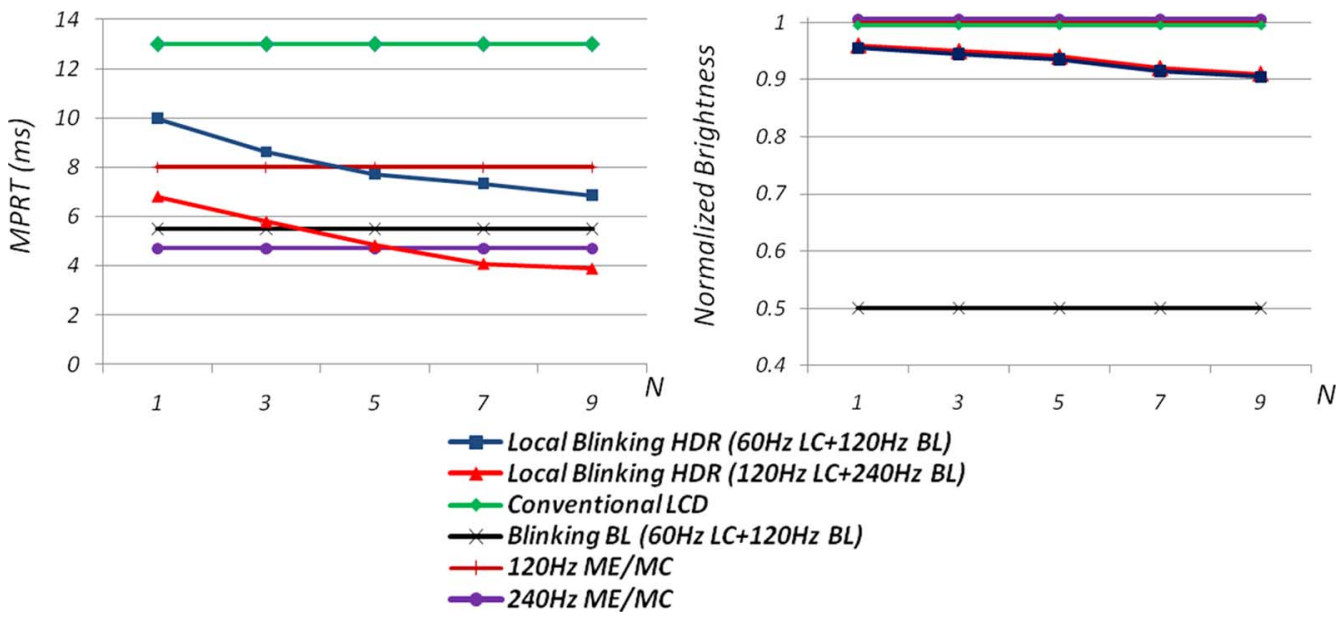

Fig. 5. Performance of MPRT and normalized brightness by different $N$-values in Local Blinking HDR LCD System and that of difference display systems.

trinsic Hold-type driving. By comparing the results of other two Impulse-type display system, Local Blinking HDR provided a sharp edge on the object and maintained the image brightness as well as the target image because of the local image compensation. For the Blinking BL part, although the results showed a better performance in Motion Blur than that of conventional LCD, the whole image brightness was reduced so that the image details were almost disappeared. If there was no substitution of high power light source, the target brightness was hardly achieved, even applying global image compensation.

\section{N-BET ANALYSIS OF LOCAL BLINKING HDR LCD SYSTEM}

Actually, N-BET of Local Blinking HDR LCD System not simply depended on the pixel gray levels, was also affected by the BL operation. Fig. 7 shows the $8 \times 8$ sets of gray to gray N-BET of Local Blinking HDR LCD System with the optimized $N=7$ value driven to $120 \mathrm{~Hz} \mathrm{LC}$ with $240 \mathrm{BL}$ frame-rate. The N-BET was not uniform over the whole gray-level rang but much more obviously suppressed in the low and middle gray levels. In Local Blinking HDR LCD System, if the original BL signals are determined to high and low gray level re- gions, the driving signals of two BL frames will also be mapped to high and low driving values which also means the blinking effect is not obvious and the performance of motion-blur suppression is low. On the other hand, if the original BL signals are in the middle region, the contrast between two mapped BL frames will be much higher, which would provide a high performance in Motion-Blur. So the original BL determination affected the performance of Motion-Blur in Local Blinking HDR LCD System. If the original BL determination attempted to get higher driving values according to the image information, the low and middle gray level image had better performance in Motion-Blur but high gray level image didn't because the low contrast of these two BL frames.

\section{IMAGE QUALITY AND ERROR QUANTIFICATION}

To investigate the image quality of Local Blinking HDR LCD system, 9 test images performed by Local Blinking and conventional HDR LCD system were evaluated and image distortion between the results and target images are quantified by CIEDE2000. CIEDE2000 was designed for predicting the visual difference for large isolated patches and often desired to 


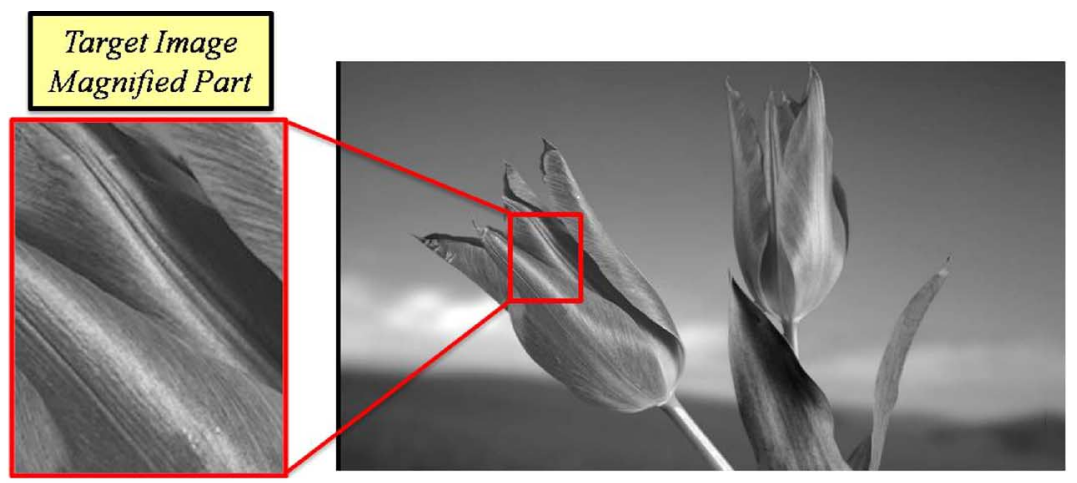

(a)

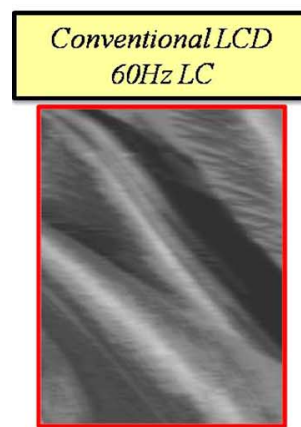

(b)

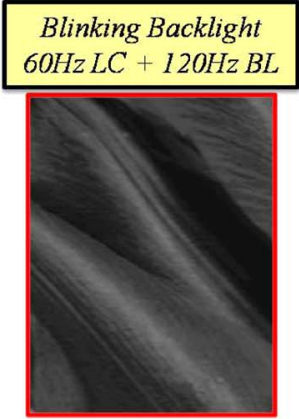

(c)

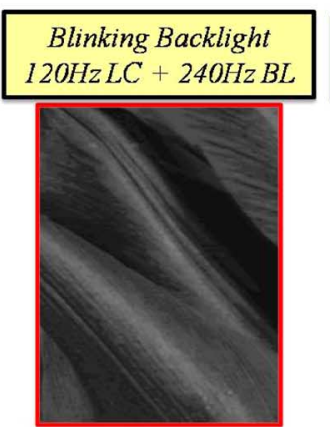

(d)

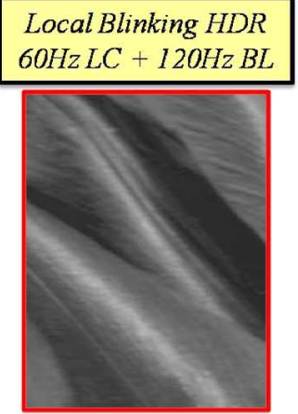

(e)

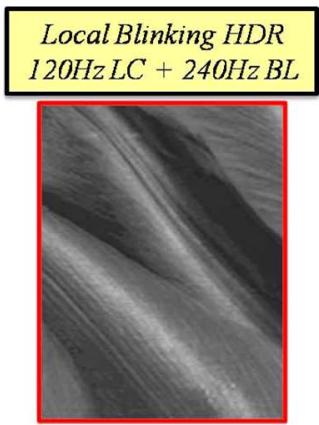

(f)

Fig. 6. Simulation results of: (a) test image displayed by (b) conventional LCD. (c), (d) Blinking Backlight systems $(60 \mathrm{~Hz} \mathrm{LC}+120 \mathrm{~Hz} \mathrm{BL}$ and $120 \mathrm{~Hz} \mathrm{LC}+$ $240 \mathrm{~Hz}$ BL frame-rate), and (e), (f) the proposed Local Blinking HDR LCD systems (60 Hz LC + $120 \mathrm{~Hz}$ BL and $120 \mathrm{~Hz} \mathrm{LC}+240 \mathrm{~Hz}$ BL frame-rate).

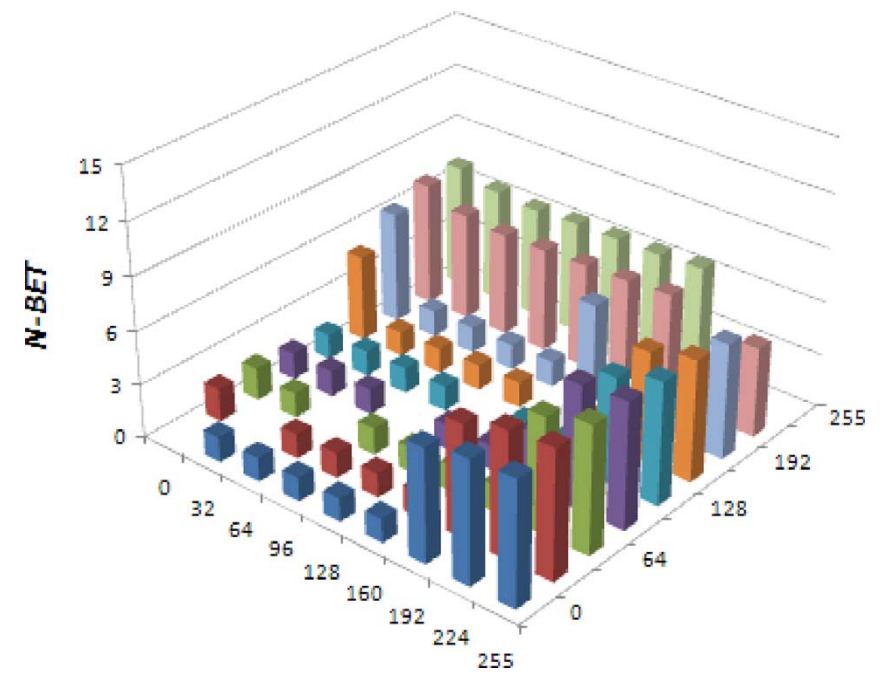

Fig. 7. N-BET of Local Blinking HDR LCD systems.

determine the perceived difference of color images [26]-[28]. Fig. 8 shows the results of color difference of optimized Local Blinking $(N=7)$ and conventional HDR LCD system to target images, and all of the original BL signals were determined by IMF algorithm. The numbers on the vertical axis are the percentage of the number of pixels which the color difference between the results of BMA and convolution are larger than 3 . The color difference was acceptable under less than 3 of CIEDE2000 which was concluded by our human experiments. The numbers on the horizontal axis indicates the corresponding images including high contrast ratio, high color saturation, and compli- cate images. Through the results of calculating the color difference, most of the images were acceptable and only a small number of pixels are distinguished the difference. The result also showed Local Blinking HDR LCD System yielded a similar image quality to that of convention one.

\section{CONCLUSION}

The proposed Local Blinking HDR LCD System provided a significant reduction in Motion Blur phenomenon and very less reduction in image brightness. In addition, Local Blinking HDR LCD System also maintained the intrinsic benefit of HDR-LCD 


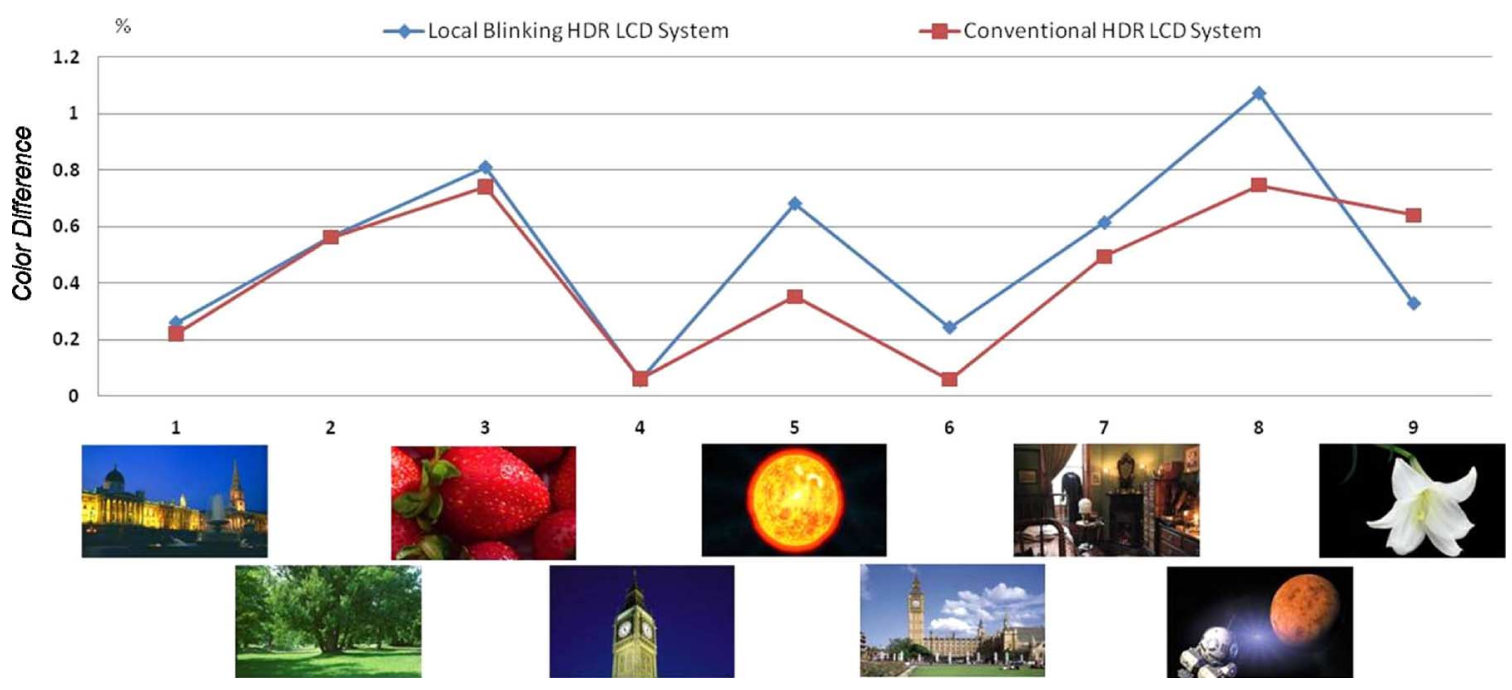

Fig. 8. Color difference of Local Blinking and conventional HDR LCD systems to target images by CIEDE2000, and the corresponding test images.

system, such as high dynamic range and low power consumption. This novel Impulse-type display system is different from the prior arts, such as Blinking Backlight system, and Scanning Backlight system, which might suffer from the suppression of image brightness. In this study, as the operated frequency was driven to $120 \mathrm{~Hz}$ LC with $240 \mathrm{~Hz}$ local blinking BL frame-rate, MPRT was reduced to $3.89 \mathrm{~ms}$ and image brightness was maintained to around $90 \%$. Moreover, the LC had not to be operated at $240 \mathrm{~Hz}$ thus we avoided the issues of slow LC response time, high data rate and complicate IC circuit. Through N-BET analysis, this method extremely lowered MPRT level to less than $3 \mathrm{~ms}$ for middle and low gray level images. Moreover, the image quality also had very less distortion compared to conventional HDR Display. Consequently, the Local Blinking HDR System could be one of the candidates for future high quality display.

\section{ACKNOWLEDGMENT}

The author would like to acknowledge S.-C. Yeh, AU Optronics Corporation (AUO), Hsinchu, Taiwan, for their support on the 37-in RGB LED Backlight HDR-LCD TV.

\section{REFERENCES}

[1] T. Kurita, "Moving picture quality improvement for hold-type AM-LCDs," in SID Symp. Dig. Tech. Papers, 2001, pp. 986-989.

[2] S. Hong et al., "Advanced method for motion-blur reduction in LCDs," in SID Symp. Dig. Tech. Papers, 2005, pp. 466-469.

[3] K. Kawabe and T. Furuhashi, "New TFT-LCD driving method for improved moving picture quality," in SID Symp. Dig. Tech. Papers, 2001, pp. 998-1001.

[4] B. W. Lee et al., "Reducing gray-level response to one frame: Dynamic capacitance compensation," in SID Symp. Dig. Tech. Papers, 2001, pp. $1260-1263$.

[5] H. X. Zhao et al., "Overdrive LUT optimization for LCD by box motion blur measurement and gamma-based thresholding method," in SID Symp. Dig. Tech. Papers, 2008, pp. 117-120.

[6] Y. W. Fang et al., "A novel algorithm for over-drive optimization," in SID Symp. Dig. Tech. Papers, 2009, pp. 709-712.

[7] S. S. Kim et al., "Novel 120-Hz TFT-LCD motion-blur-reduction technology with integrated motion-compensated frame-interpolation timing controller," J. Soc. Inf. Display, vol. 16, no. 3, pp. 403-413, 2008.

[8] S. S. Kim et al., "World's first $240 \mathrm{~Hz}$ TFT-LCD technology for full-HD LCD-TV and its application to 3D display," in SID Symp. Dig. Tech. Papers, 2009, pp. 424-427.
[9] J. I. Hiralata et al., "Super TFT-LCD for moving picture images with the blinking backlight system," in SID Symp. Dig. Tech. Papers, 2001, pp. $990-993$

[10] T. Nose et al., "A black stripe driving scheme for displaying motion picture on LCDs," in SID Symp. Dig. Tech. Papers, 2001, pp. 994-997.

[11] H. Seetzen et al., "High dynamic range display systems," SIGGRAPH 2004, ACM Trans. Graphics, vol. 23, no. 3, pp. 760-768.

[12] H. Seetzen et al., "A high dynamic range display using low and high resolution modulators," in SID Symp. Dig. Tech. Papers, 2003, pp. $1450-1453$.

[13] M. D. Tisdall et al., "Comparing signal detection between novel high-luminance HDR and standard medical LCD displays," J. Display Technol., vol. 4, no. 4, pp. 398-409, Dec. 2008.

[14] Y. K. Cheng et al., "Analyses of point spread function in high dynamic range display system," in Optics and Photonics, Taiwan, 2005, Serial G-SA-X 4-3.

[15] H. Chen et al., "Locally pixel-compensated backlight dimming on LED-backlit LCD TV," J. Soc. Inf. Display, vol. 15, pp. 981-988, 2007.

[16] L. Kerofsky and S. Daly, "Brightness preservation for LCD backlight dimming," J. Soc. Inf. Display, vol. 14, pp. 1111-1118, 2006.

[17] F. Li et al., "Two approaches to derive LED driving signals for high-dynamic-range LCD backlights," J. Soc. Inf. Display, vol. 15, pp. 989-996, 2007.

[18] C. H. Chen and H. P. D. Shieh, "Effects of backlight profiles on perceived image quality for high dynamic range LCDs," J. of Display Technology, vol. 4, no. 2, pp. 153-159, 2008.

[19] Y. K. Cheng et al., "Super-Gaussian light spread function for highdynamic-range displays," in SID Symp. Dig. Tech. Papers, 2008, pp. 983-985.

[20] Y. Igarashi et al., "Summary of moving picture response time (MPRT) and futures," in SID Symp. Dig. Tech. Papers, 2004, pp. 1262-1264.

[21] G. Z. Wang et al., "Delta-color adjustment method for color controlled backlight of high dynamic range LCD-TVs," in SID Symp. Dig. Tech. Papers, 2008, pp. 768-771.

[22] Y. Igarashi et al., "Proposal of the perceptive parameter motion picture response time (MPRT)," in SID Symp. Dig. Tech. Papers, 2003, pp. $1039-1041$.

[23] F. C. Lin et al., "Dynamic backlight gamma on high dynamic range LCD TVs," J. of Display Technology, vol. 4, no. 2, pp. 139-146, 2008.

[24] L. Kerofsky et al., "Brightness preservation for LCD backlight reduction," in SID Symp. Dig. Tech. Papers, 2006, pp. 1242-1245.

[25] L. Y. Liao and Y. P. Huang, "Blur-mask approach for real-time calculation of light spreading function (LSF) on spatial modulated high dynamic range LCDs,", unpublished.

[26] Central Bureau of the CIE, Vienna, Austria, "Improvement to industrial colour-difference evaluation" CIE Pub. 142-2001, 2001.

[27] G. M. Johnson and M. D. Fairchild, "A top down description of S-CIELAB and CIEDE2000," Color Res. Appl., vol. 28, pp. 425-435, 2003. 
[28] M. R. Luo, G. Cui, and B. Rigg, "The development of the CIE 2000 colour difference formula," Color Res., to be published.

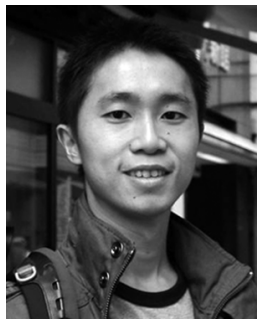

Lin-Yao Liao received the B.S. degree from Department. of Electric Engineering, National Sun Yat-sen University, Kaohsiung, Taiwan, in 2006, and is currently working toward the Ph.D. degree at Department of Photonics, Institute of Electro-Optical Engineering, National Chiao Tung University, Hsinchu, Taiwan.

His current research is including high imagequality with low power consumption LCDs, and optical design with liquid crystal lenses.

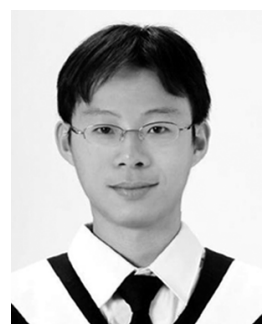

Chih-Wei Chen received the B.S. degree from Department of Mechatronics Engineering, National Changhua University of Education, Changhua, Taiwan, in 2007, and is currently working toward the Ph.D. degree from the Department of Photonics, Institute of Electro-Optical Engineering, National Chiao Tung University, Hsinchu, Taiwan.

His current research is including high imagequality LCDs, 3-D displays, and liquid crystal lens design.

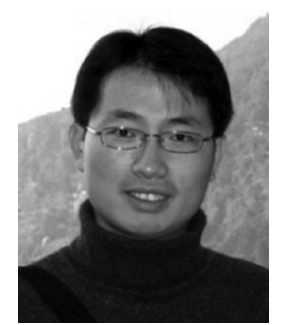

Yi-Pai Huang received the B.S. degree from National Cheng-Kung University in 1999, and the Ph.D. degree (with merit) from the Institute of Electro-Optical Engineering, National Chiao Tung University (NCTU), Hsinchu, Taiwan.

$\mathrm{He}$ is currently an assistant professor in the Department of Photonics \& Display Institute, National Chiao Tung University, Hsinchu, Taiwan. He was a Project Leader/Deputy Manager in the technology center of AUOptronics (AUO) Corporation before joining NCTU. His current research interests are advanced display systems (high dynamic range LCD and field sequential LCD), display human vision evaluation, 3-D displays, and display optics. $\mathrm{He}$ has published 18 journal papers, more than 40 international conference papers, and has 17 U.S. patents to his credit.

Dr. Huang was awarded the SID2001 Best Student Paper Award, SID2004 and SID2009 Distinguished Paper Award. He is also the secretary general of SID Taipei Chapter, and program committee of SID. 